\title{
Bony Orbital Surgery for Graves' Ophthalmopathy
}

\author{
Adrian A. Ong, MD ${ }^{1}$ Samuel DeVictor, MD ${ }^{2} \quad$ Aurora G. Vincent, MD ${ }^{1}$ Arya W. Namin, MD ${ }^{10}$ \\ Weitao Wang, MD ${ }^{3 \odot}$ Yadranko Ducic, MD, FRCS(C), FACS ${ }^{1}$ \\ 1 Otolaryngology and Facial Plastic Surgery Associates, Fort Worth, \\ Texas \\ 2 Department of Otolaryngology, Jacobs School of Medicine and \\ Biomedical Sciences, University at Buffalo, The State University of \\ New York, Buffalo, New York \\ ${ }^{3}$ Department of Otolaryngology-Head and Neck Surgery, University \\ of Rochester, Rochester, New York \\ Address for correspondence Yadranko Ducic, MD, FRCS(C), FACS, \\ Otolaryngology and Facial Plastic Surgery Associates, 923 \\ Pennsylvania Avenue, Suite 100, Fort Worth, TX 76104 \\ (e-mail: yducic@sbcglobal.net).
}

Facial Plast Surg 2021;37:692-697.

\author{
Abstract \\ Keywords \\ - orbital \\ decompression \\ - thyroid eye disease \\ - Graves' \\ ophthalmopathy
}

The majority of Graves' ophthalmopathy, or thyroid eye disease, can be managed medically; however, in refractory or severe cases, surgical intervention with orbital decompression may be necessary. The majority of the published literature is retrospective in nature, and there is no standardized approach to orbital decompression. The purpose of this review is to evaluate the various surgical approaches and techniques for orbital decompression. Outcomes are ultimately dependent on individual patient factors, surgical approach, and surgeon experience.
Graves' ophthalmopathy, or thyroid eye disease (TED), is an autoimmune condition which can affect up to $50 \%$ of patients with Graves' disease, and causes both cosmetic disfigurement and functional deficits. ${ }^{1}$ It can lead to exophthalmos, eyelid retraction and exposure keratopathy, orbital pain and pressure, diplopia, and even vision loss secondary to compressive optic neuropathy with significant impact on quality of life. Important consideration in management of patients with TED is medical management of the hyperthyroidism and comorbid cigarette dependence. Cigarette smoking has been found in many studies to increase the risk of developing TED. ${ }^{2}$ Patients with Graves' disease should be counseled about tobacco cessation in regards to both the risk of developing TED and the other well-known negative health effects of cigarette smoking. Patients with TED should also be informed that tobacco cessation may decrease the progression of the disease. The first-line treatment of Graves' disease is antithyroid drugs, while radioactive iodine and total thyroidectomy are reserved for refractory cases. ${ }^{3}$ It is recommended that patients who are treated with radioactive iodine also undergo prophylactic oral steroid treatment to help mitigate the risk of TED development. ${ }^{4}$ However, in patients presenting with TED, thyroidectomy and radioactive iodine have not been shown to affect the natural course of TED. ${ }^{5}$ Initial treatment during the acute phase of TED includes supportive medical management, and typically includes topical lubrication, head elevation, corticosteroids, and orbital radiotherapy. ${ }^{6}$ If patients develop acute vision-threatening orbital compression or enter remission and seek to restore normal globe position, surgical intervention is considered. The purpose of this manuscript is to review the role of bony orbital decompression in the management of TED.

\section{Pathophysiology of Thyroid Eye Disease}

The pathophysiology of TED is not fully understood; however, it is speculated to be caused by autoantibodies to the orbital connective tissue. ${ }^{7}$ Thyroid stimulating hormone receptor autoantibodies, also known as long-acting-thyroid stimulators (LATS), have been shown to be involved in the etiology of Graves' disease and are thought to be responsible for the development of hyperthyroidism. ${ }^{3}$ A correlation between severity of elevation of these LATS and the development of TED has been described. ${ }^{4}$ There are two phases to the disease: an active phase and quiescent phase. During the active phase, lymphocytes infiltrate the orbital tissue with subsequent fibroblast activation, release of proinflammatory published online September 9, 2021
Issue Theme Skeletal Facial Plastic Surgery; Guest Editor: Yadranko Ducic, MD, FRCS(C), FACS (c) 2021. Thieme. All rights reserved. Thieme Medical Publishers, Inc., 333 Seventh Avenue, 18th Floor, New York, NY 10001, USA
DOI https://doi.org/ 10.1055/s-0041-1735638. ISSN 0736-6825. 
cytokines, and deposition of glycosaminoglycans. There is increased production of hyaluronan, which accumulates within the orbit leading to edema and orbital soft-tissue volume expansion. The orbital fibroblasts may then differentiate into adipocytes or myofibroblasts and may explain the heterogeneity in clinical presentation. ${ }^{8}$ Ultimately, fibrosis of the extraocular muscles and fat hypertrophy occurs. The active phase waxes and wanes for many years, and is followed by a quiescent phase with no further progression of disease. Rarely, patients may experience flare-ups of their disease burden with further inflammation.

\section{Indications for Surgical Decompression}

In the majority of patients with TED, conservative management is sufficient to alleviate symptoms and surgical intervention is avoided; however, approximately $20 \%$ of patients will undergo surgical intervention. ${ }^{9}$ Indications for urgent orbital decompression include visual loss from compressive optic neuropathy or corneal exposure. Delayed decompression usually occurs after a euthyroid state is achieved and an interval of 6 months has passed assuring surgical intervention during the quiescent phase of disease. Indications for delayed decompression include asymmetric or severe proptosis, moderate-to-severe extraocular muscle hypertrophy, diplopia, ocular pain, and ocular hypertension. ${ }^{10}$ Orbital decompression should take place prior to any strabismus and/or eyelid surgery. ${ }^{11,12}$

\section{Surgical Approaches to Bony Decompression of the Orbit}

Bony orbital decompression is performed in the operating room under general anesthesia. Intravenous antibiotics and corticosteroids are administered preoperatively, and depending on the surgical approach, surgical navigation can be set up at this time. After the induction of general anesthesia, local anesthesia based on surgeon's preference is administered at the planned incisions. There are four bony boundaries of the orbit; however, only three are routinely decompressed: the orbital floor, medial wall, and lateral wall. Depending on the degree of decompression needed, a single wall or a combination of walls can be decompressed. Most commonly, the medial orbital wall is addressed first, followed by the orbital floor. If additional decompression is needed, then the lateral wall is decompressed. The patient is reassessed by ophthalmology, and if further decompression is needed, albeit rarely used, the orbital roof is decompressed. The surgical approach for each wall is discussed below.

\section{Medial Orbital Wall}

The medial orbital wall can be approached in one of two ways: transorbital or transnasal. The transorbital approaches include a transcutaneous approach using a Lynch incision and a transcaruncular approach; however, the transnasal endoscopic approach has become the most widely utilized approach to the medial orbital wall due to its many advantages.

The transnasal endoscopic approach has garnered more attention recently as it provides a direct approach to the medial wall with the advantage of no external incisions (-Fig. 1). This approach is typically performed by otolaryngologists with experience in endoscopic anterior skull base surgery. In addition, the introduction of surgical navigation technology in endoscopic endonasal surgery provides enhanced efficacy and safety. ${ }^{13,14}$ The nasal mucosa is decongested, and local anesthetic is infiltrated. After medialization of the middle turbinate, uncinectomy is performed. A total ethmoidectomy is completed to identify the lamina papyracea and create space for the orbital contents after decompression. Orbital decompression is performed by removal of the entire lamina papyracea with incision into the periorbita such that the orbital contents are herniated into the ethmoid sinuses. The periorbita is incised with a sickle knife beginning posteriorly and extending the incision anteriorly. The endoscopic transnasal approach provides better visualization and safer access to the posterior medial wall for

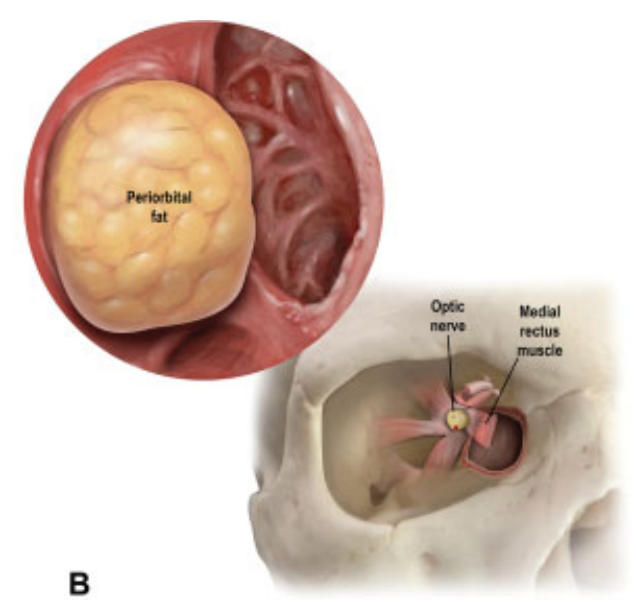

Fig. 1 Endoscopic approach to the medial orbital wall. (A) After ethmoidectomy, the lamina papyracea is removed to expose the periorbita. The periorbita is incised. (B) After incision of the periorbita, the orbital fat is prolapsed into the middle meatus. Care is taken to prevent injury to the medial rectus. 
decompression. If needed, the optic nerve can be decompressed via this technique. If the optic nerve is to be decompressed, a wide sphenoidotomy is performed and the fovea ethmoidalis is identified posteriorly. Within the sphenoid, the carotid artery and optic nerve are identified. The thick bony overlying the junction between the orbital apex and the sphenoid sinus, also known as the optic tubercle, is then removed. This bone is thick and requires the use of a diamond drill. The bone overlying the optic nerve within the sphenoid sinus is then removed, and this bone is thinner and can be removed with a Freer elevator. Once the bone has been removed off the orbital apex and optic nerve, the optic nerve sheath is then incised keeping in mind the ophthalmic artery is found in the posteroinferior quadrant of the nerve. Complications of the transnasal endoscopic approach are low, and are minimized by intimate endoscopic anatomical knowledge and experience with the technique. ${ }^{15}$ Care is taken to avoid injury to the anterior and posterior ethmoid bundle which can retract into the orbit and cause a retrobulbar hematoma. Additionally, during incision into the periorbita, the medial rectus is identified as injury to this structure can cause diplopia.

\section{Orbital Floor}

The orbital floor can be approached transorbitally using a transconjunctival incision and transantrally using the Caldwell-Luc approach.

The transconjunctival approach utilizes an incision in the palpebral conjunctiva of the lower eyelid inferior to the tarsus with two potential routes: retroseptal or preseptal. The retroseptal approach provides direct access to the infraorbital rim and after incision of the periorbita, exposure of the orbital floor is performed in usual fashion. The preseptal approach begins with eversion of the lower eyelid to identify the tarsal plate. The incision is made below the tarsus and the preseptal space is entered. With direct visualization of the septum, blunt dissection is carried down to the infraorbital rim, and the orbital floor is exposed as previously described. The transconjunctival approach can also be combined with a lateral canthotomy with inferior cantholysis, termed the "swinging eyelid" approach, to visualize both the orbital floor and lateral orbital wall, and has been described extensively for orbital decompression. ${ }^{16-18}$

The transantral approach avoids a lower eyelid incision, and affords widest exposure to the orbital floor (-Fig. 2). ${ }^{19}$ The maxillary sinus is accessed via a Caldwell-Luc approach. An incision is made in the gingivobuccal sulcus above the canine fossa and carried down to the anterior maxillary wall. The periosteum is elevated and the infraorbital nerve is identified. After entering the maxillary sinus, the mucosa is stripped and the orbital floor can be removed. The periorbita is incised away from the infraorbital nerve and allows the periorbital fat to herniate into the maxillary sinus. The anterior ethmoid air cells can also be removed to expose the medial orbital wall through this approach. This approach includes a risk of paresthesia and anesthesia due to injury to the infraorbital nerve, oroantral fistula, and devitalization of maxillary teeth.

Regardless of approach, decompression of the orbital floor is limited to removal of the bone medial to the infraorbital nerve, which limits the risk of paresthesia and anesthesia of the cheek and upper lip. In addition, a strut of bone is left anteriorly to minimize the risk of hypoglobus.

\section{Lateral Wall}

The lateral wall is typically approached by transcutaneous incisions, including the lateral eyelid crease and lateral canthotomy with inferior cantholysis (-Fig. 3). The "swinging eyelid" approach may also be utilized if the orbital floor is to be decompressed as well. The lateral one-third of an upper blepharoplasty incision is made and dissection down to the lateral orbital rim is performed. Cantholysis is then
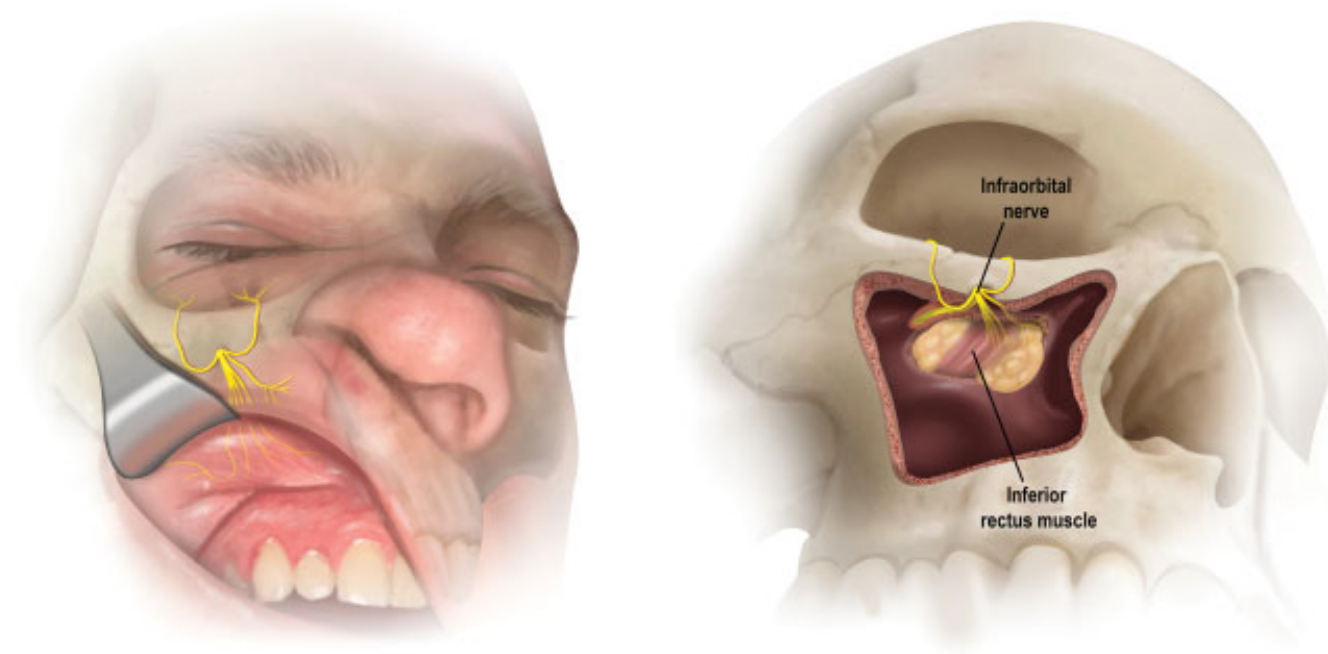

A

B

Fig. 2 Caldwell-Luc approach to the orbital floor. (A) Incision is made in the gingivobuccal sulcus. The infraorbital nerve is outlined. (B) The anterior maxillary wall is removed to expose the orbital floor. The orbital floor is removed, periorbita is incised, and orbital fat prolapses into the maxillary sinus. The infraorbital nerve and inferior rectus are outlined. 


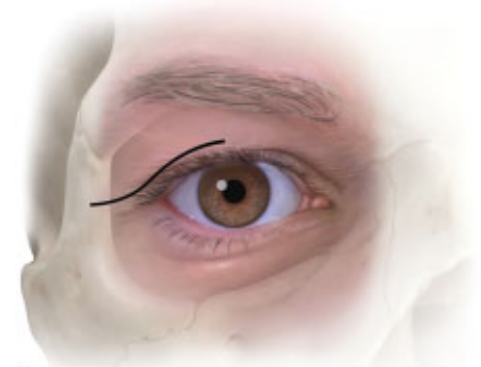

A

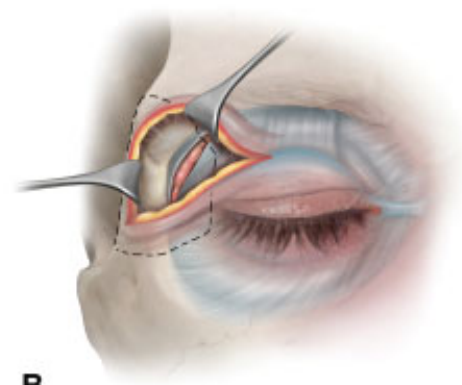

B

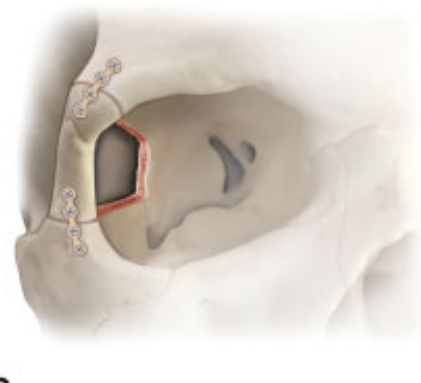

C

Fig. 3 Lateral upper blepharoplasty approach to the lateral orbital wall. (A) The incision of the lateral upper blepharoplasty is outlined. (B) The lateral orbital rim is removed en bloc. (C) Zygomatic bone and greater wing of sphenoid are removed to the suture line and allow prolapse of orbital fat into the expanded space. The lateral orbital rim is replaced and secured with mini-plates.

performed, and with a cutting burr, the lateral orbital rim is excised and placed into saline. The lateral periorbita is bluntly dissected away from the lateral wall and the orbital contents are retracted medially. The orbit is expanded laterally by drilling the lateral orbital wall until the temporalis muscle is exposed. The dissection is taken posteriorly through the greater wing of the sphenoid bone into the marrow space with or without exposure of the dura. After adequate bone removal, the periorbita is incised above and below the lateral rectus to allow the orbital contents to herniate into the expanded bony space. The lateral orbital rim is then replaced, secured with miniplates, and canthoplasty is performed to resuspend the lateral canthal tendon. Complications of this approach include cerebrospinal fluid leak secondary to dural violation, anesthesia secondary to injury to the zygomaticotemporal and zygomaticofacial neurovascular bundles, and oscillopsia.

\section{Orbital Roof}

A superior blepharoplasty or sub-brow incision is used to approach the orbital roof ( - Fig. 4). Soft tissue dissection is then carried forth to the superior orbital rim, taking care to preserve the supraorbital neurovascular bundle. A subperiorbital dissection is then carried forth along the orbital roof, and the greater wing of the sphenoid and frontal bone posterior to the frontal sinus can be removed with Freer or number nine periosteal elevator. The dura is identified and preserved during this procedure. Like the other approaches, the periorbita is incised and orbital fat can herniate the expanded bone region.

\section{Outcomes}

The primary goal of delayed orbital decompression for TED is improvement in proptosis. The choice in which wall to decompress is both individualized to the patient and dependent on surgeon comfort with technique. Likewise, the degree of decompression is dependent on surgical technique, and can be enhanced with the addition of orbital fat decompression. Single-wall decompression rarely occurs in the contemporary management of TED due to the limited reduction in proptosis compared with two-wall or three-wall decompression. If a single-wall approach is chosen, some have advocated for a deep lateral wall decompression which can reduce proptosis by 4 to $6 \mathrm{~mm} .^{20-22}$ Jefferis et al compared both their personal experience with orbital decompression and a literature review on the different approaches. Their study reported a $4.2 \mathrm{~mm}$ mean reduction with single-wall and between 2.9 and $7.6 \mathrm{~mm}$ mean reduction for a combination of two- or three-wall decompression. ${ }^{23}$ The literature review revealed a mean single-wall decompression of 3.6 to $4.8 \mathrm{~mm}$ and a mean two- or three-wall decompression of 2.5 to $8.0 \mathrm{~mm}$. When a two-wall
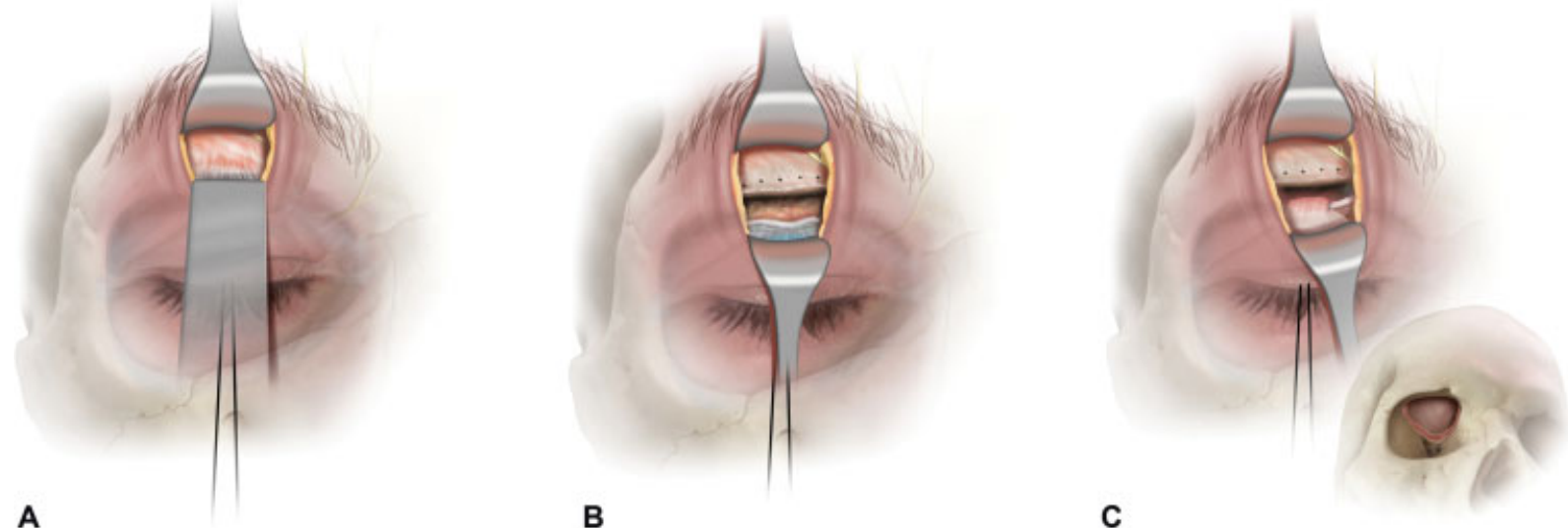

Fig. 4 Sub-brow approach to the orbital roof. (A) Exposure of the superior orbital rim. (B) Subperiosteal dissection along the orbital roof. (C) Removal of the orbital roof. After incision of the periorbita, fat can prolapse into expanded bone space. The supraorbital rim is kept intact. 
approach is chosen, surgeons may decompress the medial wall and orbital floor, termed an "inferomedial decompression," or a "balanced decompression" involving the medial and lateral orbital walls. Thapa et al showed more effective proptosis reduction with the inferomedial decompression than with the medial wall decompression alone. ${ }^{24}$ If inferomedial decompression is performed, the inferomedial orbital strut should be preserved to maintain the position of the eye. ${ }^{25}$ However, many oculoplastic surgeons have moved away from inferomedial decompression in favor of the "balanced decompression" approach. ${ }^{26}$

In recent years, there has been an increased focus on the effect of therapeutic interventions on patient-reported outcome measures. TED has a significant impact on patient quality of life, and Wickwar et al have shown approximately $26 \%$ of patients with TED having signs of clinical depression. ${ }^{27}$ A disease-specific quality-of-life questionnaire has been developed for TED called the Graves' Ophthalmopathy Quality of Life (GO-QOL) scale. With regard to orbital decompression, two studies have shown a significant impact on visual function and appearance. ${ }^{28,29}$

\section{Special Considerations}

\section{Balanced Decompression}

The "balanced decompression" involves removal of the lateral wall and medial wall while maintaining an intact orbital floor, and has become a favored technique among oculoplastic surgeons. ${ }^{26}$ The purpose is to reduce the asymmetric shift in orbital contents and thus reduce the rate of complications such as hypoglobus, new-onset diplopia, and muscle imbalance. ${ }^{30}$ Studies have shown a reduction in proptosis of approximately 4 to $6 \mathrm{~mm} .^{10,31,32}$ Additional decompression can be achieved with removal of fat.

Unal et al reported an effective reduction in proptosis with a balanced decompression with a reduced incidence of postoperative diplopia when compared with a three-wall decompression. ${ }^{33}$ However, Goldberg et al reported a lower incidence of strabismus with a lateral orbital-wall decompression compared with a balanced medial and lateral orbital-wall decompression. ${ }^{34}$ The conflicting results suggest that outcomes are likely surgeon-specific and techniquedependent.

\section{Two-Wall versus Three-Wall Decompression}

With various combinations of decompression options and preponderance of retrospective studies, there remains debate about the best approach for reducing proptosis among TED patients. Many authors report a significant improvement in proptosis reduction of approximately 7 to $8 \mathrm{~mm}$ with a three-wall decompression. ${ }^{35,36}$ However, three-wall decompression is more often associated with postoperative complications such as new-onset or worsening diplopia. ${ }^{33,36,37}$ Given the potential for increased incidence of diplopia when compared with two-wall decompression, three-wall decompression is typically reserved for patients with severe proptosis.

\section{Future Directions}

In the past, there was no specific Food and Drug Administration (FDA)-approved medical therapy for TED. Initial therapy was directed toward symptomatic management using corticosteroids, and when refractory, patients proceeded with orbital decompression. In January 2020, a monoclonal antibody to insulin-like growth factor I receptor, teprotumumab, received FDA approval specifically for the treatment of TED. In a phase 3 multicenter randomized control trial, Douglas et al showed reduction in proptosis of $2.82 \mathrm{~mm}$ in patients with active TED treated with teprotumumab compared with placebo as well as a significant improvement in GO-QOL. ${ }^{38}$ The introduction of this novel therapy will likely result in a major shift in the medical and surgical management of TED with fewer patients necessitating surgical intervention in the future.

\section{Conclusion}

TED refractory to medical management can be managed surgically with orbital decompression resulting in improved cosmesis and potential return to baseline function. There are multiple surgical techniques for orbital decompression, and the approach can be individualized based on patient characteristics and surgeon experience. Intimate knowledge of orbital anatomy and careful surgical technique can minimize complications.

\section{Note}

The work herein does not necessarily represent the views of the United States government, Department of Defense or its affiliates.

\section{Conflict of Interest}

None declared.

\section{References}

1 Bartalena L, Tanda ML. Clinical practice. Graves' ophthalmopathy. N Engl J Med 2009;360(10):994-1001

2 Thornton J, Kelly SP, Harrison RA, Edwards R. Cigarette smoking and thyroid eye disease: a systematic review. Eye (Lond) 2007;21 (09):1135-1145

3 Kim J, Choi MS, Park J, et al. Changes in thyroid stimulating hormone receptor antibody levels following total thyroidectomy or radioiodine therapy in patients with refractory Graves' disease. Thyroid 2021

4 Ponto KA, Zang S, Kahaly GJ. The tale of radioiodine and Graves' orbitopathy. Thyroid 2010;20(07):785-793

5 Bartalena L, Baldeschi L, Boboridis K, et al; European Group on Graves' Orbitopathy (EUGOGO) The 2016 European Thyroid Association/European Group on Graves' Orbitopathy Guidelines for the Management of Graves' Orbitopathy. Eur Thyroid J 2016;5 (01):9-26

6 Bartalena L, Marcocci C, Tanda ML, et al. An update on medical management of Graves' ophthalmopathy. J Endocrinol Invest 2005;28(05):469-478

7 Kazim M, Goldberg RA, Smith TJ. Insights into the pathogenesis of thyroid-associated orbitopathy: evolving rationale for therapy. Arch Ophthalmol 2002;120(03):380-386 
8 Smith TJ, Koumas L, Gagnon A, et al. Orbital fibroblast heterogeneity may determine the clinical presentation of thyroid-associated ophthalmopathy. J Clin Endocrinol Metab 2002;87(01):385-392

9 Bartalena L, Pinchera A, Marcocci C. Management of Graves' ophthalmopathy: reality and perspectives. Endocr Rev 2000;21 (02):168-199

10 Kacker A, Kazim M, Murphy M, Trokel S, Close LG. "Balanced" orbital decompression for severe Graves' orbitopathy: technique with treatment algorithm. Otolaryngol Head Neck Surg 2003;128 (02):228-235

11 Alsuhaibani AH, Carter KD, Policeni B, Nerad JA. Orbital volume and eye position changes after balanced orbital decompression. Ophthal Plast Reconstr Surg 2011;27(03):158-163

12 Mainville NP, Jordan DR. Effect of orbital decompression on diplopia in thyroid-related orbitopathy. Ophthal Plast Reconstr Surg 2014;30(02):137-140

13 Dubin MR, Tabaee A, Scruggs JT, Kazim M, Close LG. Image-guided endoscopic orbital decompression for Graves' orbitopathy. Ann Otol Rhinol Laryngol 2008;117(03):177-185

14 Prevost A, Dekeister C, Caron P, et al. Outcomes of orbital decompression using surgical navigation in thyroid-associated ophthalmopathy. Int J Oral Maxillofac Surg 2020;49(10):1279-1285

15 Antisdel JL, Gumber D, Holmes J, Sindwani R. Management of sinonasal complications after endoscopic orbital decompression for Graves' orbitopathy. Laryngoscope 2013;123(09):2094-2098

16 De Riu G, Meloni SM, Gobbi R, Soma D, Baj A, Tullio A. Subciliary versus swinging eyelid approach to the orbital floor. J Craniomaxillofac Surg 2008;36(08):439-442

17 Paridaens D, Lie A, Grootendorst RJ, van den Bosch WA. Efficacy and side effects of 'swinging eyelid' orbital decompression in Graves' orbitopathy: a proposal for standardized evaluation of diplopia. Eye (Lond) 2006;20(02):154-162

18 Sasim IV, de Graaf ME, Berendschot TT, Kalmann R, van Isterdael C, Mourits MP. Coronal or swinging eyelid decompression for patients with disfiguring proptosis in Graves' orbitopathy? Comparison of results in one center. Ophthalmology 2005;112(07): 1310-1315

19 Walsh TE, Ogura JH. Transantral orbital decompression for malignant exophthalmos. Laryngoscope 1957;67(06):544-568

20 Baldeschi L, MacAndie K, Hintschich C, Wakelkamp IM, Prummel $\mathrm{MF}$, Wiersinga WM. The removal of the deep lateral wall in orbital decompression: its contribution to exophthalmos reduction and influence on consecutive diplopia. Am J Ophthalmol 2005;140 (04):642-647

21 Ben Simon GJ, Syed HM, Lee S, et al. Strabismus after deep lateral wall orbital decompression in thyroid-related orbitopathy patients using automated hess screen. Ophthalmology 2006; 113(06):1050-1055

22 Mehta P, Durrani OM. Outcome of deep lateral wall rim-sparing orbital decompression in thyroid-associated orbitopathy: a new technique and results of a case series. Orbit 2011;30(06): 265-268

23 Jefferis JM, Jones RK, Currie ZI, Tan JH, Salvi SM. Orbital decompression for thyroid eye disease: methods, outcomes, and complications. Eye (Lond) 2018;32(03):626-636
24 Thapa S, Gupta AK, Gupta A, Gupta V, Dutta P, Virk RS. Proptosis reduction by clinical vs radiological modalities and medial vs inferomedial approaches: comparison following endoscopic transnasal orbital decompression in patients with dysthyroid orbitopathy. JAMA Otolaryngol Head Neck Surg 2015;141(04):329-334

25 Yao WC, Sedaghat AR, Yadav P, Fay A, Metson R. Orbital decompression in the endoscopic age: the modified inferomedial orbital strut. Otolaryngol Head Neck Surg 2016;154(05):963-969

26 DeParis SW, Tian J, Rajaii F. Practice patterns in orbital decompression surgery among American Society of Ophthalmic Plastic and Reconstructive Surgery members. Ophthalmol Ther 2019;8 (04):541-548

27 Wickwar S, McBain HB, Ezra DG, Hirani SP, Rose GE, Newman SP. Which factors are associated with quality of life in patients with Graves' orbitopathy presenting for orbital decompression surgery? Eye (Lond) 2015;29(07):951-957

28 Iacobæus L, Sahlin S. Evaluation of quality of life in patients with Graves' ophthalmopathy, before and after orbital decompression. Orbit 2016;35(03):121-125

29 Mourits MP, Bijl H, Altea MA, et al; European Group on Graves' Orbitopathy (EUGOGO) Outcome of orbital decompression for disfiguring proptosis in patients with Graves' orbitopathy using various surgical procedures. Br J Ophthalmol 2009;93(11):1518-1523

30 Leone CR Jr, Piest KL, Newman RJ. Medial and lateral wall decompression for thyroid ophthalmopathy. Am J Ophthalmol 1989;108(02):160-166

31 Graham SM, Brown CL, Carter KD, Song A, Nerad JA. Medial and lateral orbital wall surgery for balanced decompression in thyroid eye disease. Laryngoscope 2003;113(07):1206-1209

32 Shepard KG, Levin PS, Terris DJ. Balanced orbital decompression for Graves' ophthalmopathy. Laryngoscope 1998;108(11, Pt 1):1648-1653

33 Unal M, Leri F, Konuk O, Hasanreisoğlu B. Balanced orbital decompression combined with fat removal in Graves ophthalmopathy: do we really need to remove the third wall? Ophthal Plast Reconstr Surg 2003;19(02):112-118

34 Goldberg RA, Perry JD, Hortaleza V, Tong JT. Strabismus after balanced medial plus lateral wall versus lateral wall only orbital decompression for dysthyroid orbitopathy. Ophthal Plast Reconstr Surg 2000;16(04):271-277

35 Cansiz H, Yilmaz S, Karaman E, et al. Three-wall orbital decompression superiority to 2-wall orbital decompression in thyroidassociated ophthalmopathy. J Oral Maxillofac Surg 2006;64(05): 763-769

36 Korkmaz S, Konuk O. Surgical treatment of dysthyroid optic neuropathy: long-term visual outcomes with comparison of 2wall versus 3-wall orbital decompression. Curr Eye Res 2016;41 (02):159-164

37 Russo V, Querques G, Primavera V, Delle Noci N. Incidence and treatment of diplopia after three-wall orbital decompression in Graves' ophthalmopathy. J Pediatr Ophthalmol Strabismus 2004; 41(04):219-225

38 Douglas RS, Kahaly GJ, Patel A, et al. Teprotumumab for the treatment of active thyroid eye disease. N Engl J Med 2020;382 (04):341-352 